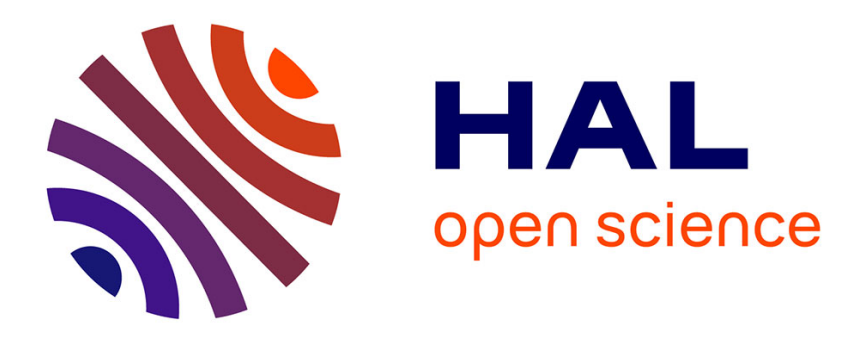

\title{
Chaleur spécifique et champ moléculaire des substances ferromagnétiques
}

\author{
Pierre Weiss, Paul-N. Beck
}

\section{To cite this version:}

Pierre Weiss, Paul-N. Beck. Chaleur spécifique et champ moléculaire des substances ferromagnétiques. J. Phys. Theor. Appl., 1908, 7 (1), pp.249-264. 10.1051/jphystap:019080070024900 . jpa-00241295

\section{HAL Id: jpa-00241295 https://hal.science/jpa-00241295}

Submitted on 1 Jan 1908

HAL is a multi-disciplinary open access archive for the deposit and dissemination of scientific research documents, whether they are published or not. The documents may come from teaching and research institutions in France or abroad, or from public or private research centers.
L'archive ouverte pluridisciplinaire HAL, est destinée au dépôt et à la diffusion de documents scientifiques de niveau recherche, publiés ou non, émanant des établissements d'enseignement et de recherche français ou étrangers, des laboratoires publics ou privés. 


\section{GHALEUR SPÉCIFIQUE ET CHAMP MOLÉCULAIRE DES SUBSTANGES FERROMAGNÉTIQUES ;}

Par MM. Pierre WEISS et PaUL-N. BECK.

\section{I}

L'hypothèse du champ moléculaire rend compte d'une manière frappante des propriétés magnétiques des corps à diverses températures, de celles des cristaux ferromagnétiques et d'un certain nombre de particularités de la courbe d'aimantation des métaux isotropes en apparence $\left({ }^{1}\right)$.

Les quantités d'énergie mises en jeu par l'aimantation et la désaimantation des substances ferromagnétiques accompagnant les variations de températures sont, par suite de l'énormité des champs moléculaires, du même ordre de grandeur que celles qui sont nécessaires à l'échauffement des corps. On sait, d'autre part, depuis les expériences fondamentales de Pionchon $\left({ }^{2}\right)$, que les chaleurs spécifiques des métaux magnétiques présentent des anomalies. Le but du présent travail est de montrer que ces anomalies correspondent quuntitativement aux variations de l'énergie magnétique.

\section{I. - partie magnétique.}

L'énergie mutuelle d'un certain nombre d'aimants de moment magnétique invariable $\mu$ est :

$$
\mathrm{E}=-\frac{1}{2} \Sigma_{1} \mathrm{H} \cos \alpha
$$

H étant le champ dans lequel est placé l'un d'entre eux et provenant de tous les autres, et $\alpha$ l'angle de $H$ avec $\mu$.

Lorsque cette somme est. étendue à tous les aimants élémentaires contenus dans 1 centimètre cube, $\mathrm{H}$ devient le champ moléculaire $\mathrm{II}_{m}$ et l'intensité d'aimantation I est la somme géométrique des moments magnétiques $\ddot{\mu}$. L'énergie d'aimantation par unité de volume est done :

$$
\mathrm{E}=-\frac{1}{2} \mathrm{IH}_{m}
$$

(1) J. de Phys., $4^{\mathrm{e}}$ série, t. VI, p. $661 ; 1907$.

(2) Proxchox, Ann. Chim. Phys., 6e série, t. XI, p. 33: 1887.

J. de Phys., $4^{\circ}$ série, t. VII. (Avril 1908.) 
ou encore, puisque le champ moléculaire est relié à l'intensité d'aimantation 1 par $\mathrm{H}_{m}=\mathrm{NI}$, où $\mathrm{N}$ est un coefficient constant :

$$
\mathrm{E}=-\frac{1}{2} \mathrm{NI}^{2}
$$

Cette énergie est négative; il faudra donc fournir de l'énergie pour désaimanter. Or I décroît d'une manière continue quand la température s'élève du zéro absolu à la température $\theta$ de disparition du ferromagnétisme spontané. La quantité totale de chaleur absorbée par le phénomène magnétique dans l'unité de masse du corps, entre la température où l'intensité d'aimantation est I et la température $\theta$, est donc :

$$
q_{m}=\frac{1}{2 J} \frac{\mathrm{N}}{\mathrm{D}} \mathbf{I}^{2}=\frac{1}{2 J} \frac{\mathrm{H}_{m} \cdot \mathbf{I}}{\mathrm{D}},
$$

où J est l'équivalent mécanique de la calorie, et $\mathrm{D}$ la densité. Et il s'ajoute à la chaleur spécifique un terme magnétique :

$$
c_{m}=\frac{1}{2 J} \cdot \frac{\mathrm{N}}{\mathrm{D}} \frac{\partial \mathrm{I}^{2}}{\partial t}
$$

L'intensité d'aimantation I étant connue en fonction de la température, il suffit de déterminer le coefficient $\mathrm{N}$. Rappelons, à cet effet, que l'on appelle constante de Curie le produit du coefficient d'aimantation spécifique \% d'un corps paramagnétique par la température absolue T. Le coefficient $\%$, lui-même, est la susceptibilité divisée par la densité. La constante de Curie C est liée à la constante N par la formule $\left({ }^{1}\right)$ :

$$
\mathrm{C} \cdot \mathrm{N} \cdot \mathrm{D} \doteq \theta \text {. }
$$

Or les trois substances étudiées ne sont pas toutes connues à l'état paramagnétique (état du fer $\gamma$ ), mais la constante de Curie dont la connaissance est nécessaire peut encore se déduire du coefficient d'aimantation spécifique $\chi^{\prime}$ à l'état de ferromagnétisme sollicité par le champ extérieur (état $\beta$ ). En effet $\left(^{1}\right)$ :

$$
\chi^{\prime}(\mathbf{T}-\theta)=\mathbf{C} .
$$

Pour ce qui est du terme $\frac{\partial I}{\partial t}$, la théorie fondée sur le champ molé-

(1) Loc. cit., p. 686 . 
culaire qui donne la variation de 1 en fonction de la température est d'accord, exactement, avec les résultats de l'expérience pour la magnétite. Pour le fer et le nickel, les résultats expérimentaux ont seulement la même physionomie générale. Réservant pour une publication ultérieure la discussion, pour chaque substance, du degré d'approximation de la théorie, dans la région $\alpha$, nous nous servirons ici des valeurs expérimentales de l. Pour le fer, nous les emprunterons à Pierre Curie; pour le nickel et la magnétite, nous aurons recours aux expériences nouvelles faites par l'un de nous. Ces expériences ne sont pas encore définitives, mais elles sont déjà plus précises que les mesures thermiques auxquelles il s'agit de les comparer.

Nous déduirons de ces expériences les quantités de chaleur $q_{m}$ nécessaires pour la désaimantation depuis les diverses températures auxquelles l'intensité d'aimantation a été observée jusqu'à $\theta$, et ensuite la valeur moyenne $\frac{\Delta q_{m}}{\Delta t}$ du terme magnétique de la chaleur spécifique correspondant aux intervalles de températures entre deux mesures successives. Ces intervalles étant suffisamment petits, on aura ainsi une image assez approchée du terme magnétique de la chaleur spécifique vraie.

Fer. - On a, d'après les expériences de Curie, des valeurs quelque peu divergentes de la constante de Curie. J'adopte une valeur moyenne $C=0,034\left(^{1}\right)$. La température $\theta$ est $756^{\circ}+273^{\circ}$ et $D=7,86$, d'où :

$$
\mathrm{N}=\frac{736^{\circ}+273^{\circ}}{0,034 \cdot 7,86}
$$

et le champ moléculaire, pour l'intensité d'aimantation trouvée par Curie égale à $216,3 \times 7,86=1700$ à la température ordinaire :

$$
\mathrm{H}_{m}=\mathrm{NI}=6530000 \text { gauss. }
$$

L'énergie de désaimantation par unité de masse est:

$$
\frac{1}{2} \times 6530000 \times 216,3=70,6 \times 10^{7} \mathrm{ergs}
$$

(1) Loc. cit., p. 685 et suiv., il a été donné par erreur pour toutes les constantes de Curie, tant expérimentales que calculées, une valeur dix fois trop faible. Les conclusions n'en sont pas altérées, sauf pour la valeur numérique du champ moléculaire, dix fois trop grande. 
et

$$
q_{n}^{20^{\circ}}=\frac{70,6}{4,19}=16,8 \mathrm{cal}
$$

En adjoignant à ce nombre ceux de la deuxième colonne du tableau I, empruntés au tableau XII de Curie('), on a calculé la troisième et la quatrième colonne du tableau I.

\begin{tabular}{|c|c|c|c|c|}
\hline$t^{\circ}$ & $\frac{I}{D}$ C. G.S. & $q_{m}$ cal. & $c_{m}=\frac{\Delta q_{m}}{\Delta t}$ & dans lintervalle \\
\hline $20^{\circ}$ & 216,3 & 16,8 & & \\
\hline $270^{\circ}$ & $207, \ddot{3}$ & $1 \ddot{3}, \ddot{j}$ & $0,00 \check{ }$ & $20^{\circ}-273^{\circ}$ \\
\hline $477^{\circ}$ & 189,6 & 12,9 & 0,013 & $273^{0}-477^{0}$ \\
\hline $601^{\circ}$ & 164,0 & 9,7 & 0,026 & $477^{\circ}-601^{\circ}$ \\
\hline $698^{\circ}$ & 127,0 & $\breve{8}, 8$ & 0,043 & $601^{\circ}-688^{\circ}$ \\
\hline $720^{\circ}$ & 100,7 & 3,6 & 0,068 & $688^{\circ}-720^{\circ}$ \\
\hline $740^{\circ}$ & 64,0 & 1,8 & 0,108 & $720^{\circ}-740^{\circ}$ \\
\hline $744^{\circ}, 6$ & 50,1 & 0,9 & 0,136 & $740^{\circ}-744^{\circ}, 6$ \\
\hline $733^{\circ}$ & $0 \mathrm{pc}$ & olation & & \\
\hline
\end{tabular}

Donc, à la température ordinaire, la chaleur spécifique n'est altérée par le phénomène magnétique que de 1 : 20 de sa valeur, tandis que dans le voisinage de $\theta$ l'appoint magnétique est de 0,136 ; ce terme disparaît brusquement à $\theta^{\circ}$. Tout se passera donc comme si la chaleur spécifique était supérieure à ce qu'elle est pour une substance non magnétique, et plus rapidement croissante, pour subir ensuite une discontinuité brusque de - 0,136 la ramenant à la valeur normale à la température 0 .

Nous sommes conduits, par cette discussion, à la conception d'un phénomène thermique nettement différent de la chaleur de fusion. Tant que les phénomènes thermiques ne sont pas serrés de très près, les denx genres de transformation ne sont pas aisés à distinguer. Les courbes de M. Osmond ( $\left.{ }^{2}\right)$, représentant l'inverse de la vitesse de refroidissement en fonction de la température, se prêtent très bien à la découverte des transformations, mais elles peuvent s'interpréter aussi bien dans l'hypothèse d'une chaleur latente absorbée à une température déterminée que dans celle d'une diminution brusque de la chaleur spécifique.

Nickel. - Les expériences de Curie se rapportent seulement à

(1) P. Curie, Ann. Chim. Phys., 7º́rie, t. V, p. $289 ; 1893$.

(2) Mémorial de l'Artillerie de la Marine, 1888. 
l'état $\beta$. On peut en déduire la température $\theta$ en remarquant que $\frac{1}{\%}$ varie proportionnellement avec $\mathrm{T}-\theta$, et l'on trouve, au moyen des nombres du tableau XV de Curie, $\theta=376^{\circ}+273^{\circ}$. De ce même tableau on déduit :

$$
\%^{\prime}=13,3 \times 10^{6} \quad \text { à } \quad \mathrm{T}=800^{\circ}+273^{\circ},
$$

d'où :

$$
\begin{aligned}
& \mathrm{C}=\varkappa^{\prime}(\mathrm{T}-0)=0,00.744 \\
& \mathrm{~N}=\frac{\mathrm{v}}{\mathrm{C} \times \mathrm{D}}=12700,
\end{aligned}
$$

en admettant, pour la densité, $\mathrm{D}=8,8$. Le champ moléculaire à la température ordinaire, où $\mathrm{I}=\check{\jmath} 00$, est :

$$
\mathrm{NI}=6330000 \text { gauss, }
$$

et, par suite, la quantité de chaleur à fournir pour la désaimantation entre la température ordinaire et la température $\theta$ :

$$
q_{m}=4,3 \mathrm{cal} .
$$

\begin{tabular}{|c|c|c|c|c|c|}
\hline \multirow{4}{*}{$\begin{array}{l}22^{\circ}, 9 \\
100^{\circ} \\
187^{\circ}\end{array}$} & \multirow{2}{*}{$\begin{array}{l}\text { If (unite arbitr.) } \\
260,75\end{array}$} & \multirow{2}{*}{$\begin{array}{c}q_{\text {in cal. }} \\
4,3\end{array}$} & \multirow{2}{*}{$\epsilon_{m}=\frac{\Delta g_{m}}{\Delta t}$} & \multicolumn{2}{|c|}{ dans l'intervalle } \\
\hline & & & & & \\
\hline & $23 \breve{3}, \breve{3}$ & 3,89 & $0,00 \leftrightarrows$ & $22^{\circ}$, & $9-100^{\circ}$ \\
\hline & $19 \%, 0$ & 2,22 & 0,008 & $100^{\circ}$ & $-187^{\circ}$ \\
\hline $247^{\circ}$ & 192,6 & 2,32 & 0,012 & $18 i^{\circ}$ & $-247^{\circ}$ \\
\hline $291^{\circ}$ & 110,7 & 1,82 & 0,016 & $247^{0}$ & $-291^{\circ}$ \\
\hline $336^{\circ}$ & วั8,13 & 0,96 & $0,0 \perp 9$ & $291^{\circ}$ & $-336^{\circ}$ \\
\hline $338^{\circ}, 5$ & 26,7 & $0,4 \dot{4}$ & 0,023 & $336^{\circ}$ & $-358^{\circ}$, \\
\hline $369^{\circ}, 3$ & 9,9 & $0,16 \breve{3}$ & 0,023 & $338^{\circ}$, & $\breve{u}-369^{\circ}, \ddot{u}$ \\
\hline $376^{\circ}$ & 0 par & golation & & & \\
\hline
\end{tabular}

Le tableau II a été établi avec ce nombre et nos nouvelles mesures de I ( ${ }^{2}$, colonne 2$)$,

Tablatau II. Nickel.

Magnétite. - Les expériences de Curie commencent, pour la magnétite comme pour le nickel, à la région $\beta$. On en déduit (tableau XIX de Curie) :

$$
\theta=336^{\circ}+273^{\circ}, \quad \text { et à } \mathrm{T}=600^{\circ}+273^{\circ}, \quad \%^{\prime}=73,2 \times 10^{-6},
$$


d'où

$$
\begin{aligned}
& \mathrm{C}=\chi^{\prime}(\mathbf{T}-\theta)=0,00468 \\
& \mathbf{N}=\frac{\theta}{\mathrm{C} \cdot \mathrm{D}}=33200,
\end{aligned}
$$

en admettant, pour la densité, $\mathrm{D}=\tilde{\mathrm{o}}, 2$. L'intensité d'aimantation à saturation à la température ordinaire étant $\mathrm{I}=430$ :

$$
\mathrm{NI}=14300000 \text { gauss. }
$$

Pour tous les échantillons de magnétite que l'un de nous a examinés, $\theta$ était voisin de $588^{\circ}+273^{\circ}$; l'identité avec la matière étudiée par Curie est donc douteuse. mais une nouvelle série de mesures dans la région $\beta$ nous a donné :

$$
\mathrm{NI}=14600000 \text { gauss, }
$$

qui concorde d'une manière inespérée avec la valeur tirée des expériences de Curie. On en déduit :

$$
q_{m}=14,4 \mathrm{cal} .
$$

\begin{tabular}{|c|c|c|c|c|}
\hline$t$ & $I^{2}$ (unité arbitr.) & $q_{m}$ cal. & $c_{m}=\frac{\Delta q_{m}}{\Delta t}$ & dans l'intervalle \\
\hline $15^{\circ}, 2$ & $4 \div 9,2$ & 14,4 & & \\
\hline $100^{\circ}$ & 418,8 & 12,6 & 0,021 & $1 \breve{5}^{\circ}, 2-100^{\circ}$ \\
\hline $187^{\bullet}, 3$ & $3 \breve{4} 4,8$ & 10,7 & 0,022 & $100^{\circ}-187^{\circ}, 3$ \\
\hline $269^{\circ}$ & 294,2 & 8,8 & 0,022 & $187^{\circ}, 3-269^{\circ}$ \\
\hline $333^{\circ}$ & 233,5 & 7,0 & 0,022 & $269^{\circ}-333^{\circ}$ \\
\hline $436^{\circ}, \mathrm{\jmath}$ & $168, \mathrm{~s}$ & $\breve{3}, 1$ & 0,023 & $333^{\circ}-436^{\circ}, \ddot{3}$ \\
\hline $488^{\circ}, 7$ & 123,5 & 3,7 & 0,026 & $436^{\circ}, 3-488^{\circ}, 7$ \\
\hline $513^{\circ}, 4_{4}$ & 97,5 & 2,9 & 0,032 & $488^{\circ}, 7-313^{\circ}, 4$ \\
\hline $536^{\circ}, \breve{a}$ & $70, \ddot{3}$ & 2,1 & 0,03ə̆ & $513^{\circ}, 4-5336^{\circ}, 5$ \\
\hline $562^{\circ}$ & 39,8 & 1,2 & 0,036 & $536^{\circ}, 5-562^{\circ}$ \\
\hline $567^{\circ}$ & 33,3 & 1,0 & 0,039 & $562^{\circ} \quad-\check{2} 67^{\circ}$ \\
\hline $572^{\circ}$ & 26,6 & 0,8 & 0,040 & $56 \pi^{\circ} \quad-572^{\circ}$ \\
\hline $577^{\circ}$ & 18,6 & 0,56 & 0,048 & $572^{\circ}-577^{\circ}$ \\
\hline 588 & 0 par ex & polation & & \\
\hline
\end{tabular}

Le tableau III a été établi avec ce nombre et nos nouvelles mesures de 1 ( ${ }^{2}$, colonne 2).

Tableau III. - Magnétite. 


\section{iI. - Partie Thermique.}

C'est l'apparition d'un coude brusque dans la courbe des chaleurs spécifiques moyennes entre $17^{\circ}$ et $t^{\circ}$ de la magnétite et, par conséquent, d'une discontinuité dans les chaleurs spécifiques vraies, qui a suggéré l'évaluation magnétique de cette discontinuité.

Pour contrôler, au moyen d'expériences calorimétriques, les résultats magnétiques que nous avons exposés ci-dessus, il est nécessaire que, dans le voisinage de la perte du ferromagnétisme spontané, les expériences soient suffisamment rapprochées pour fixer le détail du phénomène. Parmi les expériences antérieures à ce travail (') sur la chaleur spécifique des métaux magnétiques aux diverses températures, celles de Pionchon sur le fer sont seules assez complètes. Celles de Pionchon sur le nickel et le cobalt, celles de Tilden sur le nickel donnent trop peu de points dans le voisinage de la température $\theta$; celles de Harker sur le fer, assez exactes sans doute dans l'ensemble, présentent des erreurs accidentelles assez grandes pour rendre impossible la détermination des chaleurs spécifiques pour de petits intervalles.

Malheureusement l'échelle des températures de Pionchon est différente de celle de nos expériences magnétiques et thermiques dans lesquelles les couples thermoélectriques ont été étalonnés avec les points fixes de Holborn et Day. Nous avons donc fait une étude calorimétrique nouvelle du fer, du nickel et de la magnétite. Pour utiliser néanmoins les expériences si soignées de Pionchon, nous avons apporté à ses températures une correction fondée sur la comparaison des chaleurs totales entre $0^{\circ}$ et $t^{\circ}$, pour le fer dans ses expériences et dans les nôtres. Avecle degré d'approximation inhérent à ce genre d'opérations, on peut admettre que les échelles sont identiques jusqu'à $256^{\circ}$, qu'ensuite, entre $236^{\circ}$ et $750^{\circ}$, la correction à ajouter est proportionnelle à l'excès de la température sur $22^{\circ}$ et atteint $48^{\circ}$ à $750^{\circ}$. A partir de cette température, la correction est constante; elle concorde avec l'écart entre le point de fusion de l'argent de Pionchon $\left(907^{\circ}\right)$ et celui de J. Violle $\left(954^{\circ}\right)$ et de Holborn et Day $\left(935^{\circ}\right)$.

Cette correction, linéaire dans l'intervalle qui comprend la trans-

(1) Pionchon, Ann. Chim. Phys., 6 série, t. XI, p. 33 ; 1887 (Fer, Nickel, Cobalt); - Tilden, Phil. Trans., A, t. CGI, p. 37 ; 1903 (Nickel) ; - HАrker, Phil. Mag., $6^{\text {e }}$ série, t. X, p. $430 ; 1905$ (Fer). 
formatiun magnélique, réduit donc dans un rapport constant toutes les chalcurs spécifiques de cet intervalle, et comporte par conséquent un minimum d'arbitraire.

Tablead IV. - Fer (Pionchon).

\begin{tabular}{|c|c|c|c|c|}
\hline$t^{\circ}$ (Pionchon) & $t^{o}$ corrigé & $q$ cal. & $c=\frac{\Delta q}{\Delta t}$ & dans l'intervalle \\
\hline $98^{\circ}, 3$ & $98^{\circ}, 3$ & 11,1 & 0,1128 & $0-98^{\circ}, 3$ \\
\hline $308^{\prime \prime}, 2$ & $313^{\circ}, 9$ & 37,82 & 0,1239 & $98^{\circ}, 3-313^{\circ}, 9$ \\
\hline $491^{\circ}, 6$ & $315^{\circ}, 2$ & 66,97 & 0,1448 & $313^{\circ}, 9-515^{\circ}, 2$ \\
\hline $535^{0}, 5$ & $563^{\circ}, 4_{4}$ & 74,67 & 0,1597 & $515^{\circ}, 2-563^{\circ}, 4$ \\
\hline $376^{\circ}, \pi$ & $608^{\circ}, 6$ & $8:, 49$ & 0,1730 & $563^{\circ}, 4-608^{\circ}, 6$ \\
\hline $607^{\circ}$ & $641^{\circ}, 8$ & $8 \overline{8}, 46$ & 0,1798 & $608^{\circ}, 6-641^{\circ}, 8$ \\
\hline $618^{\circ}, 3$ & $634^{0}, 3$ & 90,71 & 0,1814 & $641^{\circ}, 8-634^{\circ}, 3$ \\
\hline $636^{\circ}, 4$ & $674^{\circ}, 1$ & 94,40 & 0,1864 & $634^{0}, 3-674^{0}, 1$ \\
\hline $647^{0}, 3$ & $686^{\circ}, 0 \ddot{\jmath}$ & 96,84 & 0,2042 & $6 \tau 4^{\circ}, 1-686^{\circ}, 0$ \\
\hline $6.33^{\circ}, 2$ & $69 \dot{4}^{\circ}, 7$ & 98,34 & $0,196 \breve{3}$ & 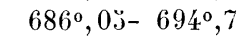 \\
\hline $666^{\circ}, 6$ & $707^{\circ}, 23$ & 101,26 & 0,2167 & $694^{\circ}, 7-707^{\circ}, 23$ \\
\hline $684^{\circ}, 2$ & $726^{\circ}, \breve{3}$ & 106,10 & 0,2322 & $707^{\circ}, 23-726^{\circ}, 53$ \\
\hline $698^{\circ}, 6$ & $742^{\circ}, 3 \ddot{3}$ & 110,33 & 0,2677 & $726^{\circ}, 3.3-742^{\circ}, 33$ \\
\hline $710^{\circ}, 6$ & 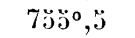 & 114,37 & 0,3072 & $742^{\circ}, 3 \ddot{\jmath}-733^{\circ}, \ddot{3}$ \\
\hline $730^{\circ}, 2$ & $777^{\circ}, 03$ & 119,90 & 0,2482 & $733^{\circ}, \ddot{3}-777^{\circ}, 0 \breve{3}$ \\
\hline $785^{\circ}, 3$ & $833^{\circ}, 3$ & 132,11 & 0,2170 & $7 \tau 7^{\circ}, 0 \Xi-833^{\circ}, 3$ \\
\hline $801^{\circ}, 4$ & $849^{\circ}, 4$ & $13 \check{,}, 99$ & 0,2410 & $833^{\circ}, 3-849^{\circ}, 4$ \\
\hline $832^{\circ}, 1$ & $880^{\circ}, 1$ & 142,70 & 0,2184 & $849^{\circ}, 4-880^{\circ}, 1$ \\
\hline $9 \ddot{30} 6^{n}, 3$ & $1004^{\circ}, 3$ & 169,16 & 0,2132 & $880^{\circ}, 1-1004^{\circ}, 3$ \\
\hline $1006^{\circ}, 1$ & $10 \preceq 4^{0}, 1$ & 180,51 & 0,2280 & $1004^{\circ}, 3-1054^{0}, 1$ \\
\hline $1056^{\circ}, 43\left(^{*}\right)$ & $1104^{\circ}, 43$ & 186,695 & 0,1228 & $1054^{\circ}, 1-1104^{\circ}, 45$ \\
\hline $1083^{\circ}, 7$ & $1131^{\circ}, 7$ & 192,54 & 0,2146 & $1104^{\circ}, 400-1131^{\circ}, 7$ \\
\hline $1115^{\circ}, 8\left(^{*}\right)$ & $1163^{\circ}, 8$ & 198,80 & 0,1638 & $11310,7-1163^{\circ}, 8$ \\
\hline $1125^{\circ}, 0$ & $1173^{\circ}, 5$ & 200,61 & 0,1866 & $1163^{\circ}, 8-1173^{\circ}, 5$ \\
\hline $1158^{\circ}, 3$ & $1206^{\circ}, 3$ & 206,51 & 0,1798 & $1173^{\circ}, \breve{b}-1206^{\circ}, 3$ \\
\hline
\end{tabular}

(*) Moyenne de deux mesures faites à des températures très voisines.

Expériences calorimétriques nouvelles (1). - Calorimètre et four électrique. - La disposition des appareils est représentée dans la fig. 1. Nous nous sommes servis du modèle ordinaire de calorimètre à eau avec enceinte à température constante. Suivant la quantité de chaleur à mesurer, il contenait des quantités d'eau variant de $\mathbf{1}^{\mathrm{kg}}, 3$ à 2 kilogrammes, de sorte que l'élévation de température restât comprise entre $1^{\circ}$ et $3^{\circ}$.

Les températures du calorimètre ont été mesurées au moyen d'un thermomètre Beckmann $\mathrm{B}$, dont l'échelle embrassait un intervalle

(1) Les expériences calorimétriques ont toutes été faites par M. Paul N.-Beck. 
de $6^{\circ}$, divisés en centièmes de degré. Ce thermomètre a été comparé avec soin à un thermomètre de même espèce muni d'un certificat de l'Institut physico-technique de Charlottenburg. Les corrections

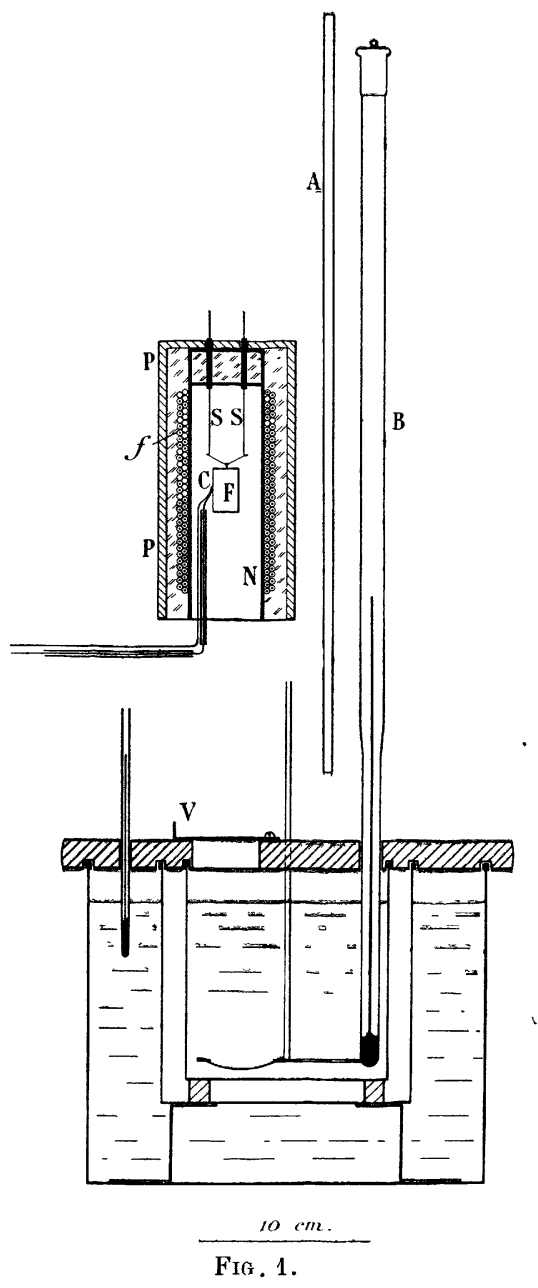

ainsi déterminées ont une marche régulière et n'ont pas dépassé un petit nombre de millièmes de degré. La tige du thermomètre a été protégée par un carton d'amiante A contre le rayonnement du four électrique, et l'on s'assurait, au moyen d'un deuxième thermomètre placé dans le voisinage, de l'invariabilité de sa température. 
La correction de refroidissement du calorimètre a été faite en observant la marche du thermomètre $B$ avant et après l'opération. Elle a toujours été très faible, la durée de l'échange de chaleur n'était que de une minute environ pour le nickel et la magnétite. Pour le fer elle a été, au début, de deux minutes et a atteint à la fin six minutes, lorsque, à la suite des chauffages répétés, l'enveloppe de platine se fut gonflée par un dégagement de gaz à l'intérieur.

Le four électrique est composé d'une carcasse en tôle de nickel $\mathrm{N}$, de 2 millimètres d'épaisseur, qui a pour but d'uniformiser la température grâce à la bonne conductibilité du métal ; le nickel a été choisi à cause de son inaltérabilité. Le four est fermé à sa partie supérieure par un double fond. Le tube de nickel est recouvert d'une lame de mica sur laquelle est enroulé bifilairement, en deux couches, un fil de nickel $f$ de 1 millimètre, recouvert d'une tresse d'amiante. La résistance électrique est à froid de 1,2 $\omega$. La protection thermique est complétée par une couche de magnésie en'poudre maintenue par le vase poreux de pile $P$ et du carton d'amiante.

La mesure des températures se faisait au moyen d'un couple thermoélectrique platine-platine rhodié $\mathrm{C}$, de $0^{\mathrm{mm}}, 6$, placé au contact du corps F. Les lectures étaient faites sur un millivoltmètre de Siemens et. Halske portant, en outre, une graduation de $0^{\circ}$ à $1000^{\circ}$. Cet instrument a été étalonné au moyen des points fixes suivants :

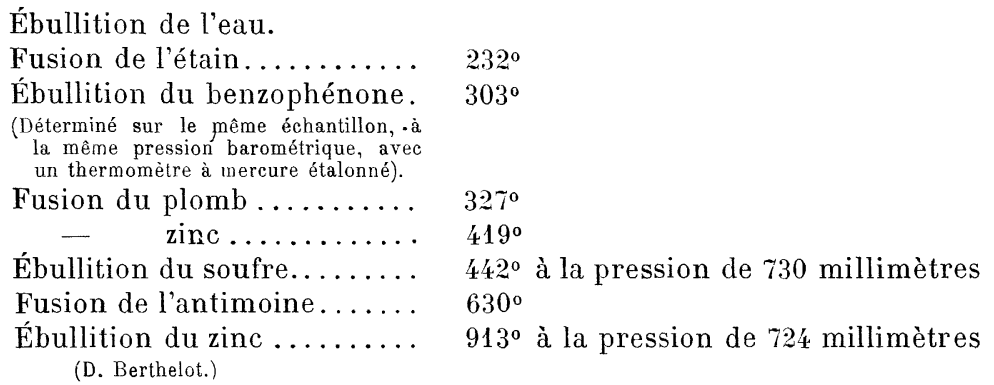

La correction a atteint une valeur maxima de 230 microvolts, c'est-à-dire environ $23^{\circ}$. Elle était sensiblement proportionnelle à la quantité à mesurer, ce qui indique que la divergence provenait surtout d'une résistance du couple plus grande que celle pour laquelle l'instrument avait été construit. Le tableau suivant montre 
GHALEUR SPÉCIFIQUE ET CHAMP MOLÉCULAIRE 259

l'efficacité de la chemise métallique pour uniformiser la température du four.

\begin{tabular}{|c|c|c|c|c|}
\hline \multirow{2}{*}{$\begin{array}{c}\text { Distance } \\
\text { du bas du four } \\
\text { en centimètres }\end{array}$} & \multicolumn{4}{|c|}{ Courant de chauffage } \\
\hline & 2,28 amp. & $3,1 \mathrm{amp}$ & $3,7 \mathrm{amp}$ & $4,7 \mathrm{amp}$. \\
\hline 13 & $9 \ddot{\jmath}^{0}, \breve{b}$ & $193^{\circ}$ & $370^{\circ}$ & $487^{\circ}$ \\
\hline 12 & $97^{\circ}$ & $199^{\circ}$ & $377^{\circ}$ & $49^{\circ}$ \\
\hline 11 & $98^{\circ}$ & $202^{\circ}$ & $383^{\circ}$ & $507^{\circ}$ \\
\hline 10 & $99^{\circ}$ & $203^{\circ}$ & $38 \pi^{\circ}$ & $513^{\circ}$ \\
\hline 9 & $100^{\circ}$ & $207^{\circ}$ & $389^{\circ}$ & $517^{\circ}$ \\
\hline 8 & $100^{\circ}, \breve{b}$ & $207^{\circ}$ & $390^{\circ}$ & $518^{\circ}$ \\
\hline 7 & $100^{\circ}, \breve{\jmath}$ & $207^{\circ}$ & $390^{\circ}$ & $518^{\circ}$ \\
\hline 6 & $100^{\circ}, \breve{\partial}$ & $20 \pi^{\circ}$ & $389^{\circ}$ & $51 \dddot{\partial}^{0}$ \\
\hline 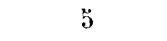 & $99^{\circ}$ & $206^{\circ}$ & $386^{\circ}$ & $308^{\circ}$ \\
\hline 4 & 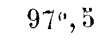 & $202^{\circ}$ & $381^{\circ}$ & $493^{\circ}$ \\
\hline 3 & $9 \ddot{3}^{\circ}, \breve{\jmath}$ & $198^{\circ}$ & $372^{\circ}$ & $481^{\circ}$ \\
\hline 2 & $93^{\circ}$ & $192^{\circ}$ & $361^{\circ}$ & $466^{\circ}$ \\
\hline 1 & $89^{\circ}$ & $180^{\circ}$ & $347^{\circ}$ & $466^{\circ}$ \\
\hline
\end{tabular}

La température a été trouvée constante dans l'étendue d'une section horizontale.

Les masses employées sont:

\begin{tabular}{lc}
\multicolumn{1}{c}{ Corps } & Enveloppes \\
$23 \mathrm{gr}, 92$ fer & $7^{\mathrm{gr}}, 36$ platine \\
$31^{\mathrm{gr}}, 2 \%$ nickel & $3^{\mathrm{gr}}, 71$ argent \\
$24 \mathrm{gr}, 73$ magnétite & $4^{\mathrm{gr}}, 31$
\end{tabular}

La correction de l'effet de l'enveloppe a une importance relative assez faible à cause de la petitesse de la chaleur spécifique du platine et à cause de la petitesse des masses d'argent. Elle a été faite, pour le platine, avec les nombres de M. J. Violle ('), et pour l'argent avec ceux de M. Pionchon (loc. cit.).

Le cylindre de fer très doux de Kohlsva (Suède) était contenu dans une envieloppe de platine de $0^{\mathrm{mm}}, 2$ d'épaisseur et fermée par un couvercle embouti et soudé. Le nickel, en petits morceaux d'environ $0^{\mathrm{cm} 3}, \check{a}$ chacun, provenait de la décomposition du nickelcarbonyle sur une paroi chaude; il ne contenait donc aucune impureté métallique. La magnétite était en plusieurs fragments provenant d'un même grand cristal de Brozzo, remarquablement homogène, étudié autrefois magnétiquement $\left(^{2}\right)$.

(1) J. VloLle, C. R., t. LXXXV, p. $543 ; 1877$.

(2) J. de Phys., $3^{\circ}$ série, t. V, p. $435 ; 1896$. 
Ces deux dernières substances étaient empaquetées dans une feuille d'argent de $0^{\mathrm{mm}}, 1$.

On s'est aperçu dès les premières expériences qu'il était nécessaire, pour obtenir des résultats concordants, de prolonger la période de chauffage à température exactement constante. Elle a duré de trois quarts d'heure à une heure et demie.

Le four électrique rayonnait une quantité de chaleur si faible que l'on a pu, sans inconvénient, le laisser en place, à une petite distance au-dessus du calorimètre, pendant toute la durée des opérations.

L'introduction du corps se faisait en ouvrant le volet $\mathrm{V}$ du calorimètre et en brûlant au moyen du courant électrique le fil fin de platine au moyen duquel la substance était suspendue aux fils de nickel S. Le corps a ainsi, au moment où il quitte le four, une vitesse appréciable. La déperdition dans le trajet entre le four et le calorimètre est donc réduite au minimum. De même la perte de chaleur par évaporation au moment où le corps traverse la surface est insensible. Le corps est reçu dans un petit panier en fil de cuivre qui sert, avec le corps lui-même, d'agitateur.

\begin{tabular}{|c|c|c|c|}
\hline$t$ & $q_{0}^{t}$ cal. & $\frac{\Delta q}{\Delta t}$ & dans l'intervalle \\
\hline 170 & 1,72 & 0,1012 & $0^{\circ}=17^{\circ}$ \\
\hline $202^{\circ}, 7$ & 23,41 & 0,1168 & $170 \quad-202^{\circ}, 9$ \\
\hline $299^{\circ}$ & 35,86 & 0,1293 & $202^{\circ}, 7-299^{\circ}$ \\
\hline $339^{6}, 7$ & 41,27 & 0,1329 & $299^{\circ} \quad-339^{\circ}, 7$ \\
\hline $396^{\circ}, 7$ & 49,31 & 0,1411 & $339^{\circ}, 7-396^{\circ}, 7$ \\
\hline $453^{\circ}$ & $57, \check{2} 2$ & 0,1438 & $396^{\circ}, 7-453^{\circ}$ \\
\hline $510^{\circ}$ & 66,15 & 0,13214 & 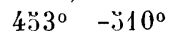 \\
\hline $533^{\circ}, 7$ & 73,06 & 0,1581 & $510^{\circ} \quad-\breve{3} 53^{\circ}, 7$ \\
\hline $598^{\circ}$ & 80,84 & 0,1756 & $533^{\circ}, 7-598^{\circ}$ \\
\hline $638^{\circ}, 7$ & 87,93 & 6,1771 & $398^{\circ} \quad-638^{\circ}, 7$ \\
\hline $691^{\circ}$ & 99,04 & 0,2124 & $638^{\circ}, 7-691^{\circ}$ \\
\hline $711^{\circ}$ & 103,41 & 0,2185 & $691^{\circ}-711^{\circ}$ \\
\hline $726^{\circ}, 7$ & 106,91 & 0,2227 & $711^{\circ} \quad-726^{\circ}, 7$ \\
\hline $741^{\circ}$ & 110,44 & 0,2469 & $726^{\circ}, 7-741^{\circ}$ \\
\hline $793^{\circ}$ & 114,20 & $0,313 b$ & $741^{\circ}-753^{\circ}$ \\
\hline $777^{\circ}, 7$ & $11 t, 41$ & 0,2280 & $733^{\circ} \quad-777^{\circ}, 7$ \\
\hline $806^{\circ}$ & 126,05 & 0,2179 & $777^{\circ}, 7-806^{\circ}$ \\
\hline $823^{3}, 7$ & 130,04 & 0,2366 & $806^{\circ} \quad-823^{\circ}, 7$ \\
\hline $844^{\circ}, 3$ & 134,78 & 0,2206 & $823^{\circ}, 7-844^{\circ}, 3$ \\
\hline $873^{\circ}, 7$ & 142,15 & 0,2503 & $844^{\circ}, 3-873^{\circ}, 7$ \\
\hline
\end{tabular}


CHALEUR SPÉCIFIQUE ET GHAMP MOLÉCULAIRE 261

Les résultats de ce tableau sont des moyennes de trois expériences faites à des températures très voisines.

Fer.

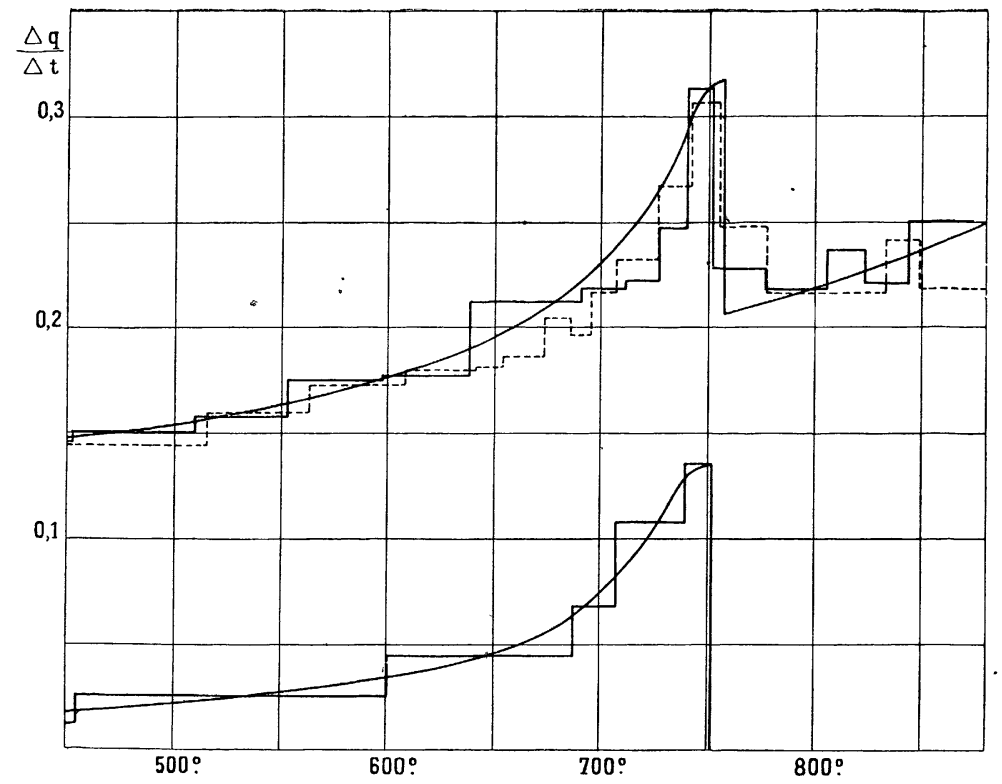

FIG. 2.

On remarquera que les valeurs maxima des chaleurs spécifiques des tablea:ux IV et $\mathrm{V}, 0,3072$ et 0,3136 , sont presque identiques et se rencontrent à la même température. Nous représentons dans la fig. 2, pour les températures de part et d'autre de $\theta$, les chaleurs spécifiques moyennes $\frac{\Delta q}{\Delta t}$ correspondant aux divers intervalles de température $\Delta t$ par des lignes horizontales, de sorte que l'aire de la ligne brisée représente les quantités de chaleur. Cette représentation manifeste avec une sensibilité extrême et les particularités du phénomène et les erreurs d'expérience.

La ligne brisée ponctuée se rapporte aux expériences de Pionchon, celle en traits pleins aux nôtres. La courbe est le tracé approximatif de la marche des chaleurs spécifiques vraies. Dans la même figure, nous avons porté en bas le terme magnétique $c_{m}$ du tableau I. On a 
donc :

$$
\begin{aligned}
& \text { magnétiquement } \\
& \theta=753+273^{\circ} \\
& c_{m}^{\theta}=0,136
\end{aligned}
$$

calorimétriquement

$$
\begin{aligned}
& \theta \frown 758+273^{\circ} \\
& c_{m}^{\theta} \simeq 0,112
\end{aligned}
$$

\begin{tabular}{|c|c|c|c|}
\hline$t$ & $q$ cal. & $\frac{\Delta q}{\Delta t}$ & dans l'intervalle \\
\hline $17^{\circ}$ & 1,66 & 0,0977 & $-17^{\circ}$ \\
\hline $123^{\circ}, 7$ & 13,5ว & 0,1124 & $17^{\circ}-123^{\circ}, 7$ \\
\hline $197^{\circ}, 7$ & 22,49 & 0,1195 & $123^{\circ}, 7-197^{\circ}, 7$ \\
\hline $247^{\circ}, \pi$ & 29,07 & 0,1316 & $197^{\circ}, 7-247^{\circ}, 7$ \\
\hline $29 \mathrm{o}^{\circ}$ & $3 \partial, 44$ & 0,1343 & $247^{\circ}, 7-295^{\circ}$ \\
\hline $318^{\circ}, 3$ & 38,69 & 0,1457 & $295^{\circ} \quad-318^{\circ}, 3$ \\
\hline $3 \% 0^{\circ}$ & 43,31 & 0,1457 & $318^{\circ}, 3-350^{\circ}$ \\
\hline $361^{\circ}$ & 44,95 & 0,1491 & $350^{\circ}-361^{\circ}$ \\
\hline $375^{\circ}, 6$ & 47,18 & 0,1527 & $361^{\circ}-375^{\circ}, 6$ \\
\hline $400^{\circ}$ & 50,25 & 0,1259 & $375^{\circ}, 6-400^{\circ}$ \\
\hline $423^{\circ}, 2$ & วั3,26 & 0,1297 & $400^{\circ}-423^{\circ}, 2$ \\
\hline $445^{\circ}, 4$ & 56,07 & 0,1261 & $423^{\circ}, 2-44 \breve{5}^{\circ}, 4$ \\
\hline $474^{\circ}, 8$ & 59,86 & 0,1290 & $445^{\circ}, 4-474^{\circ}, 8$ \\
\hline $500^{\circ}, 2$ & 63,24 & 0,1330 & $474^{\circ}, 8-500^{\circ}, 2$ \\
\hline $545^{\circ}, 8$ & 69,24 & 0,1315 & $500^{\circ}, 2-545^{\circ}, 8$ \\
\hline $599^{\circ}, 2$ & 76,32 & 0,1326 & $545^{\circ}, 8-599^{\circ}, 2$ \\
\hline $648^{\circ}, 4$ & 82,93 & 0,1344 & $599^{\circ}, 2-648^{\circ}, 4$ \\
\hline $705^{\circ}, 2$ & 90,83 & 0,1391 & $648^{\circ}, 4-70{ }^{\circ}, 2$ \\
\hline $751^{\circ}, 6$ & 97,91 & 0,1526 & $705^{\circ}, 2-751^{\circ}, 6$ \\
\hline $799^{\circ}, 6$ & $104,8 \mathrm{~J}$ & 0,1445 & $\tau 51^{\circ}, 6-\tau 99^{\circ}, 6$ \\
\hline
\end{tabular}

Nickel. - Les résultats de nos mesures sur le nickel sont consignés dans le tableau VI, qui contient des moyennes de cinq expériences à des températures très voisines.

$$
\text { TABLEAU VI. - Nickel. }
$$

La fig. 3 représente de la même manière ces expériences sur le nickel. On trouve :

$$
\begin{gathered}
\text { magnétiquement } \\
\theta=376+273^{\circ} \\
c_{m}^{\theta}=0,02 \mathrm{o}
\end{gathered}
$$

$$
\begin{gathered}
\text { calorimétriquement } \\
\theta \frown 376+273^{\mathrm{e}} \\
c_{m}^{\oplus} \backsim 0,027
\end{gathered}
$$

Magnétite. - Les résultats de nos mesures sur la chaleur spécifique dela magnétite sont consignés dans le tableau VII, qui contient des moyennes de cinq expériences à des températures très voisines. 
Nickel.

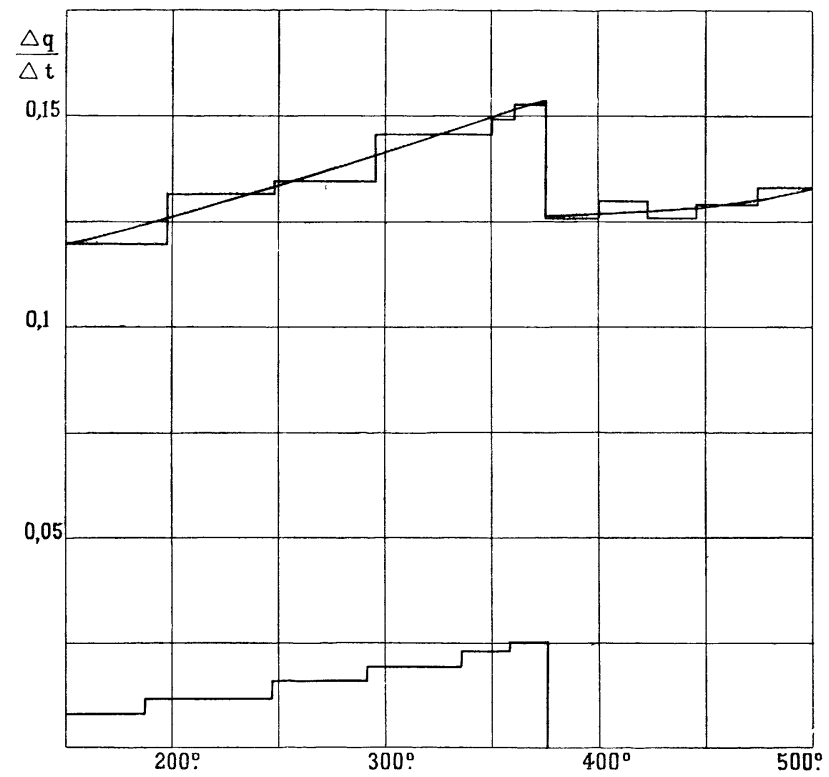

FIg. 3.

Magnétite.

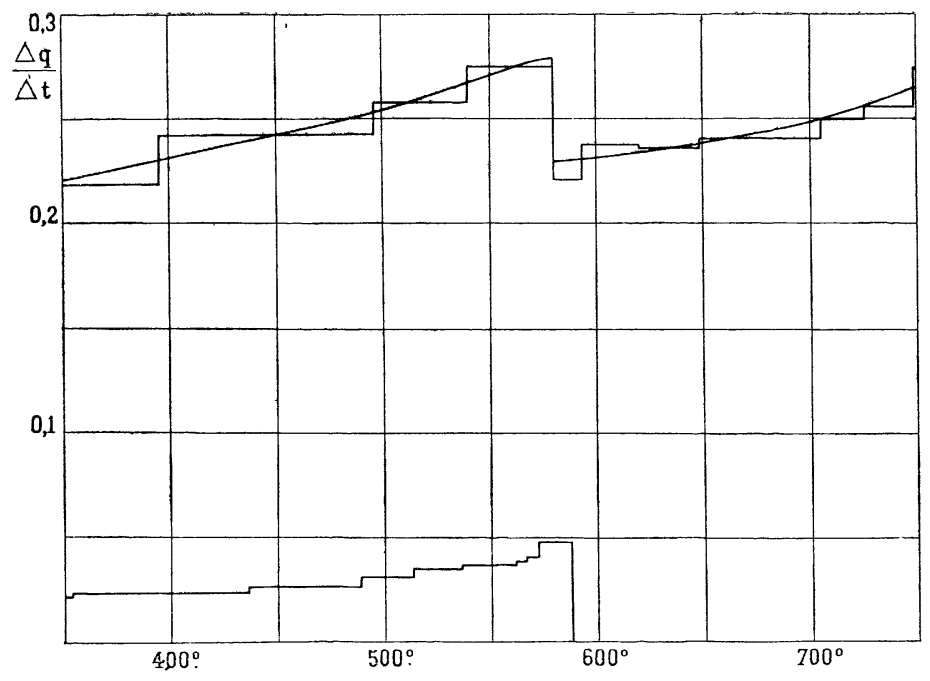

Fig. 4. 
Tableau VII. - Magnétite.

$\begin{array}{crcc}t & q_{i 1}^{t} \text { cal. } & \frac{\Delta q}{\Delta t} & \text { dans l'intervalle } \\ 17^{\circ} & 2,7 i & 0,1647 & 0^{\circ}-17^{\circ} \\ 100^{\circ}, 8 & 16,84 & 0,1679 & 17^{\circ}-100^{\circ}, 8 \\ 198^{\circ} & 33,62 & 0,1831 & 100^{\circ}, 8-198^{\circ} \\ 304^{\circ}, 4 & 55,96 & 0,2003 & 198^{\circ}-304^{\circ}, 4 \\ 394^{\circ}, 7 & 75,68 & 0,2184 & 304^{\circ}, 4-394^{\circ}, 7 \\ 496^{\circ} & 100,24 & 0,2424 & 394^{\circ}, 7-496^{\circ} \\ 540^{\circ}, 1 & 111,39 & 0,2373 & 496^{\circ}-540^{\circ}, 1 \\ 580^{\circ}, 2 & 122,80 & 0,2746 & 540^{\circ}, 1-580^{\circ}, 2 \\ 593^{\circ}, 2 & 125,30 & 0,2213 & 580^{\circ}, 2-393^{\circ}, 2 \\ 620^{\circ}, 2 & 132,02 & 0,2380 & 593^{\circ}, 2-620^{\circ}, 2 \\ 647^{\circ}, 6 & 138,47 & 0,2363 & 620^{\circ}, 2-647^{\circ}, 6 \\ 703^{\circ}, 6 & 151,94 & 0,2403 & 647^{\circ}, 6-703^{\circ}, 6 \\ 725^{\circ}, 6 & 157,42 & 0,2493 & 703^{\circ}, 6-725^{\circ}, 6 \\ 748^{\circ}, 2 & 163,21 & 0,2561 & 725^{\circ}, 6-748^{\circ}, 2 \\ 791^{\circ}, 8 & 175,17 & 0,2743 & 748^{\circ}, 2-791^{\circ}, 8\end{array}$

La $f g$. 4 représente de la même manière les expériences sur la magnétite. On a :

$$
\begin{aligned}
\text { magnétiquement } \\
\theta=588+273^{\circ} \\
c_{m}^{\oplus}=0,048
\end{aligned}
$$$$
\begin{aligned}
& \text { calorimétriquement } \\
& \theta \frown 580+273^{\circ} \\
& c_{m}^{\oplus} \simeq 0,050
\end{aligned}
$$

En résumé, pour le fer, le nickel et la magnétite, la température à laquelle se manifeste une discontinuité de la chaleur spécifique vraie coïncide avec celle de la perte du ferromagnétisme spontané, et la grandeur de cette discontinuité concorde avec celle que l'on calcule à partir des propriétés magnétiques, en s'appuyant sur l'hypothèse du champ moléculaire. 